\title{
450673 - ULTRASOUND MEASUREMENT OF PALPATED INTERCRISTAL (TUFFIER'S) LINE HEIGHT IN VOLUNTEERS
}

\author{
Christopher Pysyk, MD, Desiree Persaud, MD, Gregory Bryson, MD, Anne Lui, \\ MD

\section{Anesthesiology, University of Ottawa, Ottawa, ON, Canada}

Introduction: The intercristal line (ICL) is a common clinical landmark used to guide the level for neuraxial techniques. We sought to determine if ultrasonography of the spine, while in clinical positioning for a spinal or epidural anesthetic, confirms the classical teaching that the ICL occurs at the L3/4 intervertebral space.

Methods: After obtaining ethics approval and informed consent, non-gravid, elective surgical patients and volunteers were examined in conventional position (seated, spine flexed) for neuraxial techniques by a single observer. The ICL was identified by palpation of the iliac crests and the skin at this level was marked with erasable pen. Paramedian ultrasound (SonoSite ${ }^{\circledR}$ MicroMAXX ${ }^{\mathrm{TM}}$ 2-5 $\mathrm{MHz}$ curved array probe) identified the vertebral level at which the palpated ICL occurred. The proportion of ultrasound assessments of ICL found at L2/3, L3/4, and L4/5 were compared with Fisher's Exact test. $\mathrm{P}<0.05$ was considered significant.

Results: Thirty-three of fifty males (66.7 \%) and fifty of sixty-four females $(78.1 \%)$ had an ICL at L3/4. Six males (12.0 \%) and ten females (15.6\%) had an ICL at L4/5, P = 0.54. Eleven males (22.2 \%) and four females (6.3 \%) had an ICL at L2/3, P < 0.02. Height, age, waist circumference, mass, and body mass index were not related to ICL level in males or females.

Discussion: In clinically relevant position for neuraxial techniques, the majority of males and females had an ultrasound-measured intercristal line at L3/4. However, significantly more males than females had an intercristal line at L2/3. This should be taken into account when determining a safe needle insertion point for subarachnoid techniques given that up to $10 \%$ of the population may have a conus medullaris extending caudal to L2 (1).

References: 1 . Spine 1998 23: 1452-1456 


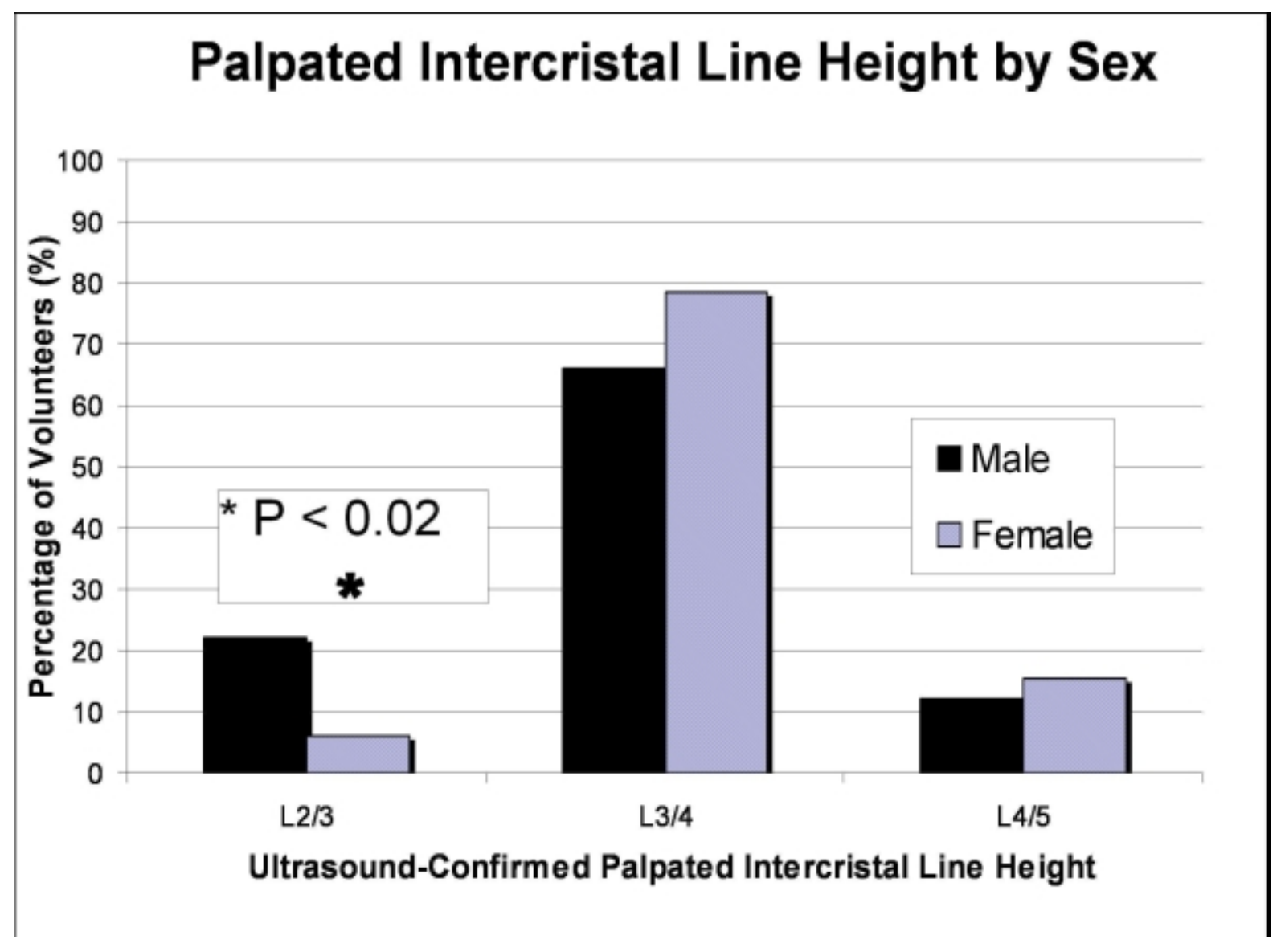

Figure 1. Distribution of the Palpated Intercristal Line Vertebral Level determined by Ultrasound in Males and Females 